\title{
Future Therapy of Cholangiocarcinoma
}

\author{
Chair: \\ Ruben R. Plentz ${ }^{\mathrm{a}}$ \\ Participants: Michael Bitzer ${ }^{\mathrm{a}} \quad$ Tim Greten $^{\mathrm{b}} \quad$ Silvio Nadalin $^{\mathrm{c}} \quad$ Arndt Vogel $^{\mathrm{d}}$ \\ a Department of Internal Medicine I, University Hospital Tübingen, Tübingen, Germany; \\ ${ }^{b}$ Center for Cancer Research, National Cancer Institute, Bethesda, MD, USA; \\ ${ }^{c}$ Department of Visceral and Transplantation Surgery, University Hospital Tübingen, Tübingen, Germany; \\ ${ }^{\mathrm{d}}$ Department of Gastroenterology, Hepatology and Endocrinology, Hanover Medical School, Hanover, Germany
}

\section{Question 1: According to your experience, is the} number of treated patients with cholangiocarcinoma rising? Please explain why, and what are the reasons for it?

Bitzer: For unclear reasons the number of intrahepatic cholangiocarcinoma seemed to increase during the last decade, whereas the incidence of extrahepatic cholangiocarcinoma decreased. Currently, no satisfying explanation exists for these observations.

Greten: The number is increasing because more cases of previously diagnosed cancers of unknown primary are now diagnosed as cholangiocarcinoma due to better imaging and pathology options.

Nadalin: In the case of 'treated' patients the answer is yes. The prognosis of cholangiocarcinoma (both intrahepatic and perihilar) without any kind of therapy is very poor. Therefore, any kind of treatment can provide better results in terms of overall survival. In this context, surgery does represent the only curative option.

Vogel: In my own experience, there is no increase of treated patients because we used to treat almost all (fit) patients in our clinic in the last decade. However, until recently, there was no established standard therapy, and patients were mainly treated with gemcitabine - similar to patients with pancreatic cancer. The ABC-02 trial provided convincing evidence for the combination of gemcitabine and cisplatin and has been the standard of care for patients with cholangiocarcinoma since 2010. I could imagine that some physicians that were more reluctant to treat cholangiocarcinoma patients felt more confident with the evidence provided by this large prospective trial.

\section{Question 2: Please explain the interdisciplinary therapeutic approach for patients with early cholangiocarcinoma at your center. How valuable is transplantation for these patients?}

Bitzer: In early cholangiocarcinoma, operability is the most important issue. Despite low 5-year survival rates even in completely resected tumors (R0 resection), surgery is currently the only possibility to cure the disease. All patients are discussed in an interdisciplinary tumor board, based on magnetic resonance imaging or computed tomography scanning and, if necessary, endoscopic retrograde cholangiopancreatography (ERCP) as an additional investigation, depending on tumor localization, performance status, and laboratory values. In selected patients, fluorodeoxyglucose-positron emission tomography (FDG-PET) scanning can contribute additional important details to the tumor staging.

Greten: Patients with cholangiocarcinoma are routinely undergoing surgery, but we rarely list these patients for transplant. Most patients present with more advanced disease.

Nadalin: First of all an interdisciplinary team should be provided. This consists mainly of: oncologist (usually subspecialized in gastrointestinal and hepatic oncology), hepatologist, radiologist (diagnostic imaging and intervention), endoscopist, radiation oncologist, anesthesiologist, and surgeon (usually subspecialized in gastrointestinal and hepatic oncology and with long-term experience in liver transplantation). Liver transplantation plays no role in intrahepatic cholangiocarcinoma due to the high and very early recurrence rate of the disease with almost no chance of long-term survival. Liver transplantation for perihilar cholangiocarcinoma plays a relevant role in selected patients treated according to the multimodal concept of the Mayo Clinic in Rochester, USA. In such

\section{KARGER}

(c) 2016 S. Karger GmbH, Freiburg

Fax +497614520714 
cases excellent overall and disease-free survival rates $(<60 \%)$ can be achieved after 5 years. This concept is based on a patient-oriented allocation system and local donation rates. In Germany, one of the last countries worldwide in terms of deceased donor organ donation, such a policy is currently missing. Therefore, deceased donor liver transplantation (DDLT) for patients with perihilar cholangiocarcinoma cannot be granted in a fashionable time. Living donor liver transplantation (LDLT) represents the only valid alternative in this regard. Due to the planning capability of the procedure, the Mayo protocol can be applied with subsequent excellent results.

Vogel: All patients with cholangiocarcinoma are discussed in our interdisciplinary tumor board before we start any treatment. We offer our patients a broad array of treatment options including chemotherapy, resection, radiofrequency ablation (RFA), transarterial chemoembolization (TACE), selective internal radiation therapy (SIRT), photodynamic therapy (PDT), radiotherapy, and chemosaturation. Currently, we rarely recommend liver transplantation for patients with cholangiocarcinoma outside of clinical trials. Based on the available evidence, I do believe that transplantation is an interesting option for selected patients with early cholangiocarcinoma, and we were happy to refer patients with early irresectable hilar cholangiocarcinoma for transplantation within the Pro-Duct study.

\section{Question 3: What is your opinion about adjuvant chemotherapy for cholangiocarcinoma? When do you perform adjuvant chemotherapy and when not? Is radiotherapy an alternative procedure?}

Bitzer: Adjuvant chemotherapy is currently not established but all patients with resected cancer should be encouraged to enter clinical trials for adjuvant therapies. Adjuvant therapy outside of studies is primarily discussed with patients having a high risk such as vascular invasion, $\mathrm{R} 1$ resection, or positive lymph nodes, and with patients of young age. However, due to a lack of data that clearly support this strategy, each indication for adjuvant therapy remains an individual decision.

Radiotherapy is currently not performed in our center in patients with this indication.

Greten: We routinely treat patients with $\mathrm{R} 1$ resection with gemcitabine (or 5-fluorouracil). We discuss this lack of data with patients who had an R0 resection. Frequently, these patients also undergo adjuvant chemotherapy [1]. Radiation treatment is sometimes used in patients with gallbladder cancer [2].

Nadalin: From my personal perspective as a surgeon, there is no prospective randomized controlled study supporting the role of adjuvant chemotherapy for cholangiocarcinoma at the moment. In the case of R1 resection and nodal involvement, however, it would make sense to me. Radiotherapy may play a role in case of perihilar cholangiocarcinoma with R1 status in the distal bile duct. Radiotherapy with a radiation field applied directly to biliodigestive anastomoses (BDA) and the resection surface may cause complications in BDA. In my personal opinion, endoscopic or transhepatic PDT may play an important role in residual bile duct with R1 status or in case of intraductal papillary neoplasia (IPN).

Vogel: Overall, the outcome of patients after resection is so poor that most of us believe that adjuvant therapy could improve survival, which is also supported by recent meta-analyses. Based on these data and until the first clinical trials are reported, we carefully discuss the possibility of adjuvant chemotherapy for (fit) patients with R0 resections, specifically patients with more advanced disease and nodal involvement, and of chemoradiotherapy for patients with $\mathrm{R} 1$ resections.

\section{Question 4: What could be the best endoscopic strategy for cholangiocarcinoma? Are PDT and RFA reasonable options, and, if yes, for which group of patients?}

Bitzer: We do not currently perform PDT or intraductal RFA in patients with cholangiocarcinoma.

Greten: We do not perform PDT; RFA is almost never used.

Nadalin: Endoscopy in perihilar cholangiocarcinoma plays a double role: i) diagnostically: through imaging (i.e. ERCP and eventually endoscopic ultrasound) as well as cytological or even better histological sampling; ii) therapeutically: PDT may play a role in Tis or T1 perihilar cholangiocarcinoma with a maximal depth of $5 \mathrm{~mm}$. This would make sense for short segmental perihilar cholangiocarcinoma of extrahepatic bile duct or IPN. I do not have any personal experience with RFA.

Vogel: One of the mainstays in the treatment of cholangiocarcinoma is to relieve obstructive jaundice through the placement of biliary stents, which has been shown to improve symptoms and to prevent infectious complications such as cholangitis. The quality of evidence for PDT and RFA is very low but the available studies indicate that there is a promising trend toward improved survival as well as improved quality of life in selected patients compared to stenting alone, despite the significant heterogeneity between the different studies. Nowadays, however, there is clear evidence for a significant survival benefit for cholangiocarcinoma patients treated with chemotherapy, and additional prospective studies are required to specifically analyze the impact of PDT/RFA on the morbidity and mortality of patients treated with systemic chemotherapy, which should be the mainstay of therapy.

Question 5: After failure of first-line chemotherapy with e.g. cisplatin and gemcitabine, do you offer patients second-line treatment? Which chemotherapeutic drugs do you select in this setting and why? 
Bitzer: Yes. If the performance status and the laboratory values allow additional systemic therapy, we offer second-line treatment, preferably in clinical studies. Outside of studies we currently prefer the FOLFIRI regimen. In patients with good performance status, however, we try to obtain additional molecular data on the tumor, including next generation sequencing and information on microsatellite stability/instability (MSI).

Greten: We only treat patients in trials in the second-line setting.

Vogel: We do not have any prospective evidence that secondline therapy improves survival after first-line therapy. However, many cholangiocarcinoma patients are still in an excellent performance status after failure of first-line therapy, and we offer these patients mainly irinotecan-based therapy similar to patients with other gastrointestinal cancers such as pancreatic or colon cancer.

\section{Question 6: Systemic therapy by SIRT might be a treatment option for patients with cholangiocarcinoma. What is your personal experience with SIRT?}

Bitzer: For well-selected patients, SIRT is a treatment alternative; however, it is currently not clear which patients benefit most from such an approach. In a retrospective multicenter study, the use of SIRT and TACE was compared in intrahepatic cholangiocarcinoma without showing a major difference regarding outcome.

Greten: We do not use SIRT for cholangiocarcinoma.

Nadalin: SIRT nowadays represents a potential palliative therapeutic option of irresectable intrahepatic cholangiocarcinoma. In this regard, no data are available on the short- and long-term results. Particular attention should be paid in case of multifocal intrahepatic cholangiocarcinoma with miliary distributions in sick livers such as fibrotic or cirrhotic ones. In such cases a SIRT may cause acute deterioration of liver function (i.e. radiation-induced liver degeneration).

Vogel: There is increasing evidence that cholangiocarcinoma are very sensitive to radiotherapy, and we consider external and internal radiotherapy as a valid option for our patients. We have seen good and bad results, and the outcome appears to be very similar to the few published, retrospective studies with a median survival of around 1 year after the first procedure.

\section{Question 7: How do you see the role of immuno- therapy in the treatment of cholangiocarcinoma?}

Bitzer: Current data support the application of immunotherapy at least for patients with mismatch repair-deficient tumors. Future studies will improve our knowledge which additional criteria allow for a reliable prediction of tumor responses or which combination therapies improve the number of patients benefitting from this approach.

Greten: There is no good data yet. Current ongoing clinical trials will show whether this may be an option in the future.

Nadalin: Very promising but not established in this context.

Vogel: Given the success in other cancers, there is of course also growing interest to use checkpoint inhibitors in cholangiocarcinoma. The few cholangiocarcinoma patients with MSI will most likely experience a similar benefit as other MSI patients. On the other hand, patients without MSI also appear to have a similar benefit like patients with other gastrointestinal cancers without MSI, with response rates below $20 \%$. Nevertheless, I do think that checkpoint inhibitors will improve survival in cholangiocarcinoma patients, though not as monotherapy but in combination with other chemo-, targeted, or local therapies.

\section{Participants}

Prof. Dr. Michael Bitzer

Abteilung Innere Medizin I

Universitätsklinikum Tübingen

Otfried-Müller-Straße 10, 72076 Tübingen, Germany

Michael.Bitzer@med.uni-tuebingen.de

Prof. Dr. Tim Greten

Center for Cancer Research

National Cancer Institute

Building 10, Room 3B43

Bethesda, MD 20892, USA

gretentf@mail.nih.gov

Prof. Dr. Silvio Nadalin, FEBS

Universitätsklinik für Allgemein-, Viszeral- und Transplantationschirurgie Universitätsklinikum Tübingen

Hoppe-Seyler-Straße 3, 72076 Tübingen, Germany

Silvio.Nadalin@med.uni-tuebingen.de

Prof. Dr. Arndt Vogel

Medizinische Hochschule Hannover

Klinik für Gastroenterologie, Hepatologie und Endokrinologie

Carl-Neuberg-Straße 1, 30625 Hannover, Germany

Vogel.Arndt@mh-hannover.de 